\title{
Virus-Induced Gene Silencing (VIGS) as a Reverse Genetic Tool to Study Development of Symbiotic Root Nodules
}

\author{
G. D. Constantin, ${ }^{1}$ M. Grønlund, ${ }^{1}$ I. E. Johansen, ${ }^{1}$ J. Stougaard, ${ }^{2}$ and O. S. Lund ${ }^{1,3}$ \\ ${ }^{1}$ Department of Genetics and Biotechnology, Faculty of Agricultural Sciences, University of Aarhus, Thorvaldsensvej 40, \\ DK-1871 Frederiksberg C, Denmark; ${ }^{2}$ Laboratory of Gene Expression, Department of Molecular Biology, University \\ of Aarhus, Gustav Wieds Vej 10, DK-8000 Aarhus C, Denmark; ${ }^{3}$ Department of Plant Biology, Faculty of Life Sciences, \\ University of Copenhagen, Thorvaldsensvej 40, DK-1871 Frederiksberg C, Denmark
}

Submitted 23 October 2007. Accepted 13 February 2008.

\begin{abstract}
Virus-induced gene silencing (VIGS) can provide a shortcut to plants with altered expression of specific genes. Here, we report that VIGS of the Nodule inception gene (Nin) can alter the nodulation phenotype and Nin gene expression in Pisum sativum. PsNin was chosen as target because of the distinct non-nodulating phenotype of nin mutants in $P$. sativum, Lotus japonicus, and Medicago truncatula. The vector based on Pea early browning virus (PEBV) was engineered to carry one of three nonoverlapping fragments (PsNinA, PsNinB, and PsNinC) derived from the PsNin cDNA. Vector inoculation was mediated by agroinfiltration and, 2 weeks later, a Rhizobium leguminosarum bv. viceae culture was added in order to induce root nodulation. At this time point, it was estimated that systemic silencing was established because leaves of reference plants inoculated with PEBV carrying a fragment of Phytoene desaturase displayed photo bleaching. Three weeks after Rhizobium spp. application, plants inoculated with a control vector nodulated normally, whereas nodulation was almost eliminated in plants inoculated with a vector carrying PsNinA and PsNinC. For plants inoculated with a vector carrying PsNinB, nodulation was reduced by at least $45 \%$. Downregulation of $P s N i n$ transcripts in plants inoculated with vectors carrying PsNin cDNA fragments was confirmed and these plants displayed a relative increase in the root/shoot ratio, as expected if nitrogen fixation had been impaired.
\end{abstract}

Additional keywords: sym35, symbiosis, Tobravirus

The number of vectors for virus-induced gene silencing (VIGS) and their applications to study gene function continues to expand (Burch-Smith et al. 2004). Studies of pathogenactivated defense pathways are especially well represented (Bhattarai et al. 2007; Liu et al. 2005; Rowland et al. 2005).

Corresponding author: I. E. Johansen; E-mail: e.johansen@dias.kvl.dk

Current address of M. Grønlund: Biosystems Department, Ris $\emptyset$ National Laboratory, Technical University of Denmark, DK-4000 Roskilde, Denmark.

* The $\boldsymbol{e}$-Xtra logo stands for "electronic extra" and indicates that two supplementary figures are published online.
However, application to studies of microbial symbioses with rhizobia or arbuscular mycorrhizal fungi is still lacking. Studies of symbiosis with nitrogen-fixating bacteria are limited to legume species that form nodules in response to Rhizobium spp. colonization (Spaink 2000). VIGS vectors have been developed for Pisum sativum (Constantin et al. 2004) and Glycine max (Zhang and Ghabrial 2006), whereas there are still no reports of efficient VIGS vectors for the main model legumes Lotus japonicus and Medicago truncatula. Earlier, P. sativum was used to characterize mutants affecting nodulation (Duc and Messager 1989); however, studies in P. sativum have been limited because of low transformation efficiency (Krejci et al. 2007) and a genome estimated to be 10 times larger than the genomes of L. japonicus and M. truncatula (Bennett and Leitch 2004).

We have shown previously that the vector based on Pea early browning virus (PEBV, genus Tobravirus) can induce a phenotype in roots, indicating that VIGS was functional in roots and that plants infected by the PEBV vector carrying a neutral fragment nodulated normally (Constantin et al. 2004). The virus vector based on Tobacco rattle virus (TRV, genus Tobravirus) also induces silencing in roots (Bhattarai et al. 2007; Valentine et al. 2004) but is not known to infect any legume species systemically (Robinson 2003). The PEBV vector consists of two binary plasmids, pCAPE1 and pCAPE2, containing cDNA of PEBV RNA1 and RNA2, respectively, placed under control of the Cauliflower mosaic virus $35 \mathrm{~S}$ promoter and nopaline synthase terminator (Constantin et al. 2004). The cDNA of PEBV RNA2 is modified to allow insertion of heterologous sequences replacing the genes required for nematode transmission (MacFarlane and Popovich 2000). Establishment of recombinant virus infection in the test plants is accomplished by agroinfiltration (Constantin et al. 2004), and this allows inoculation of many plants with high efficiency at a low cost.

To assess whether PEBV-mediated VIGS can alter expression of genes involved in nodulation to an extent that gives rise to a phenotype affecting nodule formation, we chose to target the Nodule inception gene (Nin) first described in L. japonicus (Schauser et al. 1999). In P. sativum, PsNin was characterized in sym35 mutants that have a non-nodulating phenotype similar to L. japonicus nin mutants (Borisov et al. 2003). Recently, two MtNIN mutant alleles were cloned and characterized in $M$. truncatula (Marsh et al. 2007). Nodules of L. japonicus are of the determinate type whereas $P$. sativum and $M$. truncatula 
produce indeterminate nodules. In all three species, nin mutants are characterized by excessive root hair curling, inability to form infection threads, and, consequently, failure to form any nodules. This phenotype places the function of NIN early in the nodulation process; however, several lines of evidence indicate that NIN also functions at later steps in nodulation. Nin transcripts are expressed in developing and mature nodules of L. japonicus (Schauser et al. 1999) and in meristematic cells and cells of the infection zone in indeterminate nodules of $P$. sativum (Borisov et al. 2003). Recent studies suggest that NIN is required for bacterial entry and initiation of nodule meristems developing into root nodules (Marsh et al. 2007; Tirichine et al. 2007). The L. japonicus Nin gene also was implicated in nitrate regulation of nodulation (Barbulova et al. 2007) and experiments in M. truncatula showed expression of an ENOD11 promoter GUS fusion in a larger area of the root in Mtnin mutants, suggesting that NIN is involved in regulation of nodule number (Marsh et al. 2007). Here, we describe the effect of targeting PsNin expression by VIGS mediated by a PEBV vector carrying different fragments of PsNin.

\section{RESULTS}

\section{Adaptation of the PEBV VIGS protocol to accommodate controlled symbiosis.}

To target $P s N i n$ transcripts for silencing mediated by PEBV, the procedure described previously (Constantin et al. 2004) was modified in order to allow systemic spread of virus and initiation of silencing before Rhizobium spp. inoculation of the plants (Fig. 1A). Initially, the effect of PEBV infection on nodulation was determined. One set of 6 plants did not receive PEBV inoculum and another set of 10 plants were agroinoculated with pCAPE1/pCAPE2-Con, which contained a 524-bp insert from the plant virus Bean yellow mosaic virus (Constantin et al. 2004). Two weeks later, Rhizobium leguminosarum bv. viceae was applied and nodulation was scored after another 3 weeks. Plants that did not receive PEBV inoculum on average developed 50 nodules, with an average of 24 pink nodules. Plants that had been agroinoculated with pCAPE1/pCAPE2Con on average developed 61 nodules, with an average of 30 pink nodules. This difference was not significant $(P>0.4)$, implying that PEBV infection did not cause major changes to the nodulation phenotype.

As a visible marker for the onset of gene silencing, Phytoene desaturase (PDS) transcripts were targeted for silencing in a set of reference plants that were agroinoculated with pCAPE1/pCAPE2-PDS (Constantin et al. 2004). As a control to monitor the effect of PEBV infection on the plants, a second set of reference plants was agroinoculated with pCAPE1/pCAPE2-Con. In plants agroinoculated with pCAPE1/pCAPE2-PDS, photo bleaching started to develop on leaves 12 to 14 days postinoculation. At that time point, an $R$. leguminosarum bv. viceae culture was initiated and applied a few days later. As a control for unintentional rhizobia infection, a third set of reference plants was not inoculated with Rhizobium spp. Three weeks post-Rhizobium spp. application, 30 to 150 mature pink nodules had developed on the roots of virus-uninoculated plants and plants that had been agroinoculated with pCAPE1/pCAPE2-Con, whereas plants that were not Rhizobium spp. inoculated did not develop nodules. At the same time point, photobleaching still was developing on plants agroinoculated with pCAPE1/pCAPE2-PDS. This protocol assured that Rhizobium spp. application was timed according to the establishment of VIGS in each experiment and root nodules developed to maturity along with ongoing VIGS, measured as continued bleaching of leaves on plants inoculated with pCAPE1/pCAPE2-PDS.
VIGS phenotype

of constructs carrying fragments of Ps Nin cDNA.

In legumes, mutants with lesions in the Nin gene display a distinct phenotype that makes it a suitable target to assess the feasibility of VIGS as a tool for nodulation studies. Two nonoverlapping PSNin cDNA fragments corresponding to nucleotides 1,720 to 2,149 (PsNinA) and 2,212 to 2,647 (PsNinB) of AJ493066, respectively (Borisov et al. 2003), were cloned into the PEBV RNA2 vector, thereby creating the constructs pCAPE2-PsNinA (430 bp) and pCAPE2-PsNinB (436 bp), respectively (Fig. 1B). Following the protocol described above, the nodulation phenotype was assessed in two independent experiments where 20 to 30 P. sativum plants
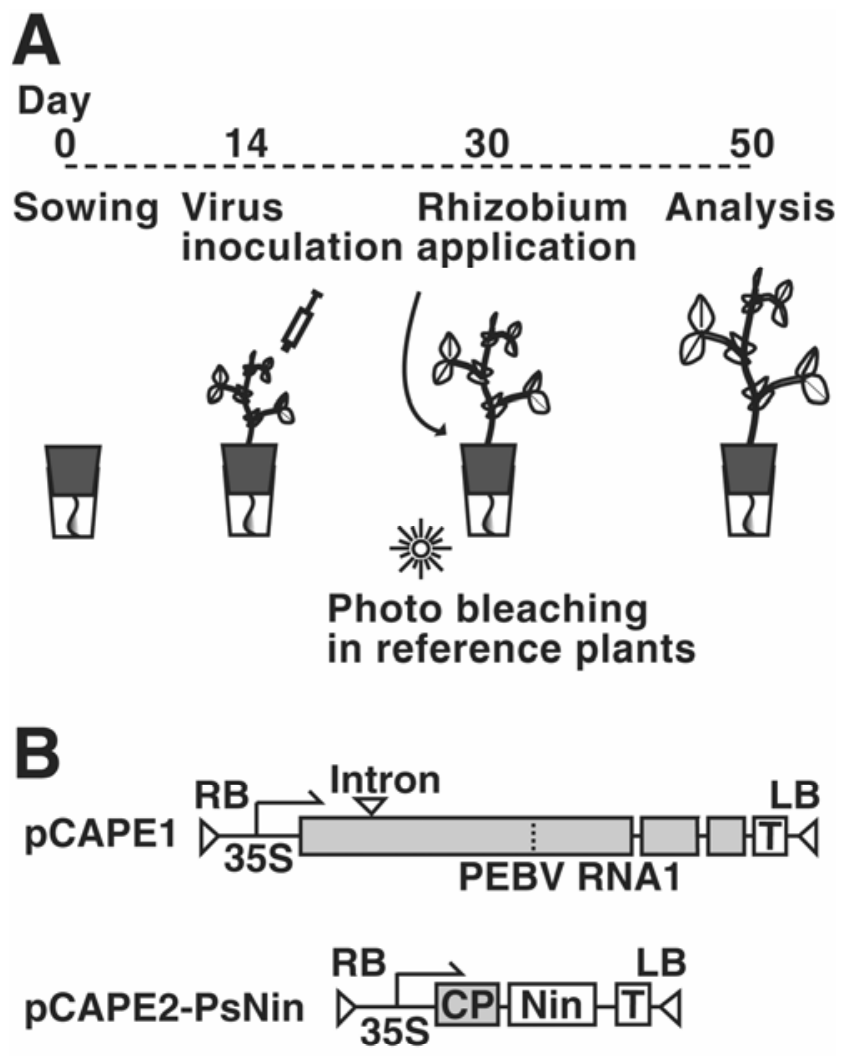

\begin{tabular}{|c|}
\hline PsNIN 1071 bp mRNA - AJ4930 \\
\hline NinC NinA NinB \\
\\
NinA253 $\square$ \\
NinA 186
\end{tabular}

Fig. 1. Application of virus-induced gene silencing to nodulation experiments in Pisum sativum. A, Seed were germinated day zero and transferred to sterile clay granules connected by a wick to a reservoir of nutrient medium without N. Two weeks after germination, plants were agroinoculated with Pea early browning virus (PEBV) vector cDNA constructs. Reference plants were agroinoculated with a construct targeting $P$. sativum Phytoene desaturase to provide a visual marker for establishment of silencing. At 12 to 14 days after agroinoculation, reference plants displayed photo bleaching and Rhizobium culture was started and applied to test plants approximately 30 days after sowing. Three weeks later, approximately 50 days after sowing, nodulation was analyzed. B, PEBV vectors with expression cassettes of RNA1 (pCAPE1) and RNA2 (pCAPE2) inserted between right and left border (RB and LB) of a binary plasmid. Transcriptional control was exerted by a $35 \mathrm{~S}$ promoter and a NOS terminator (T). pCAPE2-Nin vectors contained cDNA of PEBV RNA-2 with insertions of cDNA derived from $P$. sativum Nodule inception gene (PsNin). The origin of the inserted fragments is indicated below the box illustrating the complete mRNA sequence (AJ493066). The black bar marks the region encoding the conserved RWPRK domain (Schauser et al. 2005). 
were agroinoculated with pCAPE1 in combination with pCAPE2-PsNinA, pCAPE2-PsNinB, or pCAPE2-Con. Ten plants were inoculated with pCAPE1/pCAPE2-PDS in each experiment to time the application of Rhizobium culture according to initiation of photobleaching as described above. The total number of nodules produced per plant was counted 3 weeks after application of Rhizobium spp. The absolute number of nodules formed varied markedly between the two experiments but the relative differences between the groups of plants tested were similar (Fig. 2A). On roots of plants inoculated with pCAPE1/pCAPE2-Con, the average number of nodules

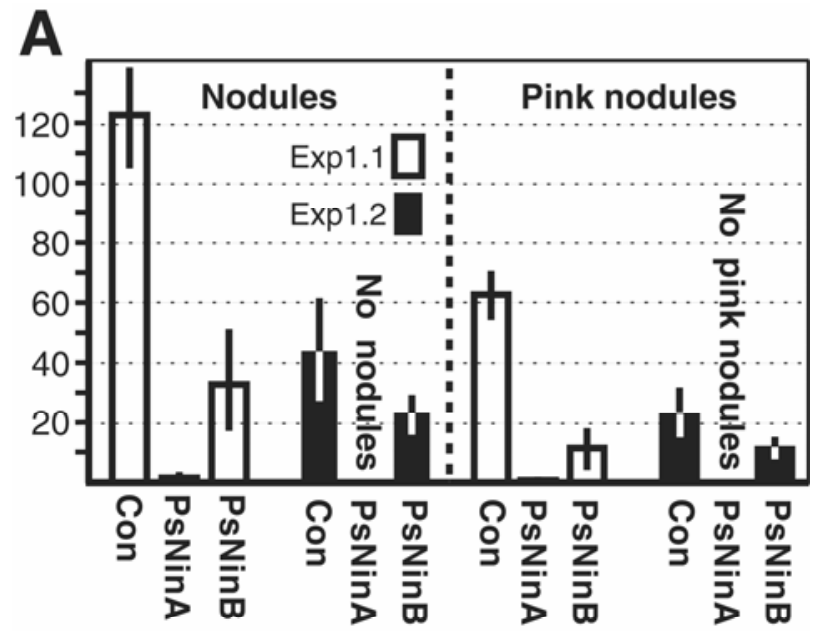

B

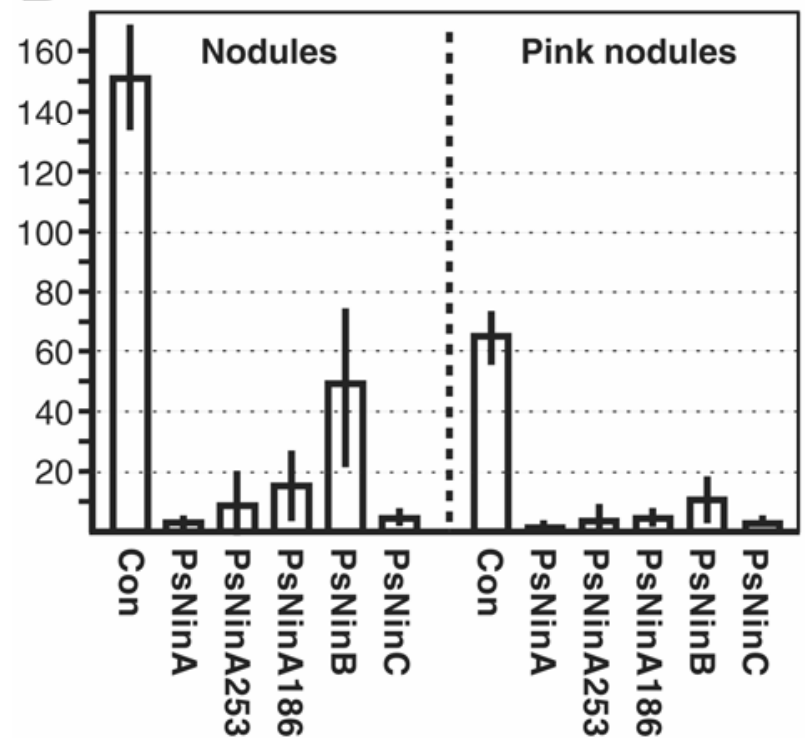

Fig. 2. Nodules developing on Pisum sativum. Columns display the average number of visible nodules per plant (left) and average number of pink nodules per plant (right). Bars represent $95 \%$ confidence intervals. A, Nodulation on roots of $P$. sativum plants agroinoculated with Pea early browning virus (PEBV) vectors carrying a fragment that was not expected to target any endogenous genes (Con), or fragments of $P$. sativum Nodule inception gene (PsNin) corresponding to nucleotides 1,720 to 2,149 (PsNinA of 430 bp) and nt 2,212 to 2,647 (PsNinB of 436 bp), respectively. The two experiments are presented separately because nodulation on control plants varied significantly between experiments $\mathbf{B}$, Nodulation on roots of $P$. sativum plants agroinoculated with PEBV vectors carrying the fragments Con, PsNinA, PsNinB, a fragment corresponding to nucleotides 106 to 538 of PsNin cDNA (PsNinC of 433 bp), a 253-bp subfragment of PsNinA corresponding to nucleotides 1,897 to 2,149 (PsNinA253), or a 186-bp subfragment of PsNinA corresponding to nucleotides 1,964 to 2,149 (PsNinA186). Results of two experiments were combined because inoculation on control plants was not considered significant between experiments $(P>0.05)$. per plant was 122 in the first experiment and 44 in the second experiment. On roots of plants agroinoculated with pCAPE1/ pCAPE2-PsNinA, an average of 0.7 nodules was formed per plant in the first experiment and 0 in the second. On roots of plants agroinoculated with pCAPE1/pCAPE2-PsNinB, nodulation was reduced $45 \%$ or more, with an average of 34 and 24 nodules in the first and second experiment, respectively (Fig. 2A). Statistical analysis confirmed that these differences were significant at $P<0.0001$ for pCAPE1/pCAPE2-Con versus pCAPE1/pCAPE2-PsNinA and for pCAPE1/pCAPE2-Con versus pCAPE1/pCAPE2-PsNinB, and at $P<0.0003$ for pCAPE1/pCAPE2-PsNinA versus pCAPE1/pCAPE2-PsNinB. Roots of all plants agroinoculated with pCAPE1/pCAPE2-Con developed both pink and white nodules whereas roots on $>75 \%$ of plants agroinoculated with pCAPE1/pCAPE2-PsNinB developed both pink and white nodules, $4 \%$ developed only white nodules, and 20\% developed no nodules. On roots of plants agroinoculated with pCAPE1/pCAPE2NinA, nodules were seen on only $15 \%$ of the plants in the first experiment.

Pink nodules can fix $\mathrm{N}$ whereas white nodules have not developed bacteroids capable of $\mathrm{N}$ fixation (Ott et al. 2005). In three experiments including only plants agroinoculated with pCAPE1/pCAPE2-PsNinB and pCAPE1/pCAPE2-Con and experiment 1.1 and 1.2 (Fig. 2A), the ratio of pink/total nodules ranged from 0.15 to 0.44 on roots of plants agroinoculated with $\mathrm{pCAPE} 1 / \mathrm{pCAPE} 2-\mathrm{PsNinB}$, whereas this ratio ranged from 0.44 to 0.62 on roots of plants agroinoculated with pCAPE1/pCAPE2-Con (Supplementary Fig. S1). This suggested that agroinoculation with pCAPE1/pCAPE2-PsNinB caused a greater reduction in pink nodules than in the total number of nodules.

\section{SiRNA Scan to predict off-target silencing induced by PsNinA and PsNinB.}

It was not expected that agroinoculation with pCAPE1/ pCAPE2-PsNinA and pCAPE1/pCAPE2-PsNinB would result in different nodulation phenotypes because the PsNin inserts were of equal size. The PsNinA fragment covered a region encoding the RWP-RK domain of NIN that is highly conserved in proteins encoded by the Nin gene family, whereas the PsNinB fragment covered a region with little or no conservation between proteins encoded by the Nin gene family (Schauser et al. 2005). Small interfering RNAs (siRNAs) derived from the trigger sequences may target transcripts of other genes (off-targets) with regions of identity or complementarity to the trigger sequences (Xu et al. 2006). It was shown that a 22-nucleotide (nt) sequence identity was sufficient to cause off-target silencing; however, genes with 21- to 22-nt identity but with one mismatch to the trigger sequence were not silenced. Therefore, it was possible that the different effect of PsNinA and PsNinB was due to off-target silencing. To predict off-targets of PsNinA and PsNinB, a sequence similarity search using the blastn program was conducted against the $\mathrm{Na}-$ tional Center for Biotechnology Information nucleotide collection and expressed sequence tag (EST) sequences from $P$. sativum. For PsNinA, the longest sequence of complete identity was $20 \mathrm{nt}$ in the gene for chalcone synthase (X80007). For PsNinB, the longest sequence of complete identity was $17 \mathrm{nt}$ in a $\beta$-galactosidase-like mRNA (DQ435678). This result probably reflected the limited sequence information available for $P$. sativum. Therefore, the corresponding Nin fragments from M. truncatula (MtNinA and MtNinB) and L. japonicus (LjNinA and $\mathrm{LjNinB}$ ) were used to scan the M. truncatula and L. japonicus datasets for targets of predicted efficient siRNAs using siRNA Scan (Xu et al. 2006). Default settings were applied to search for genes with at least $21 \mathrm{nt}$ identity or complementarity. With these settings, only MtNin and LjNin were 
identified as targets. An siRNA SCAN of PsNinA and PsNinB against the Arabidopsis dataset identified a sequence in TC255366 with 24-nt identity to PsNinA. Although these results did not predict serious off-target effects, off-target silencing cannot be ruled out. Therefore, to test whether off-target effects were responsible for the phenotype generated by PsNinA, the phenotype induced by a third fragment was analyzed because the chance that two different fragments target the same genes is low.

\section{VIGS phenotype induced by a third fragment, PsNinC,} and shorter fragments of PsNinA.

A third cDNA fragment with a size equal to PsNinA and PsNinB was cloned into pCAPE2, generating pCAPE2PsNinC (433 bp). The PsNinC fragment covered nucleotides 106 to 538 of the PsNin cDNA, which encodes the N-terminus of PsNIN (Fig. 1B) that is not conserved in proteins encoded by other members of the Nin gene family (Schauser et al. 2005). Blastn of PsNinC against the P. sativum nucleotide collection and EST did not produce any hits, and siRNA Scans of the corresponding fragments in $L$. japonicus (LjNinC) and (MtNinC) identified only LjNin and MtNin as targets with at least $21 \mathrm{nt}$ perfect match. In addition, to tEST whether the silencing phenotype induced in plants inoculated by $\mathrm{pCAPE} 1 / \mathrm{pCAPE} 2-\mathrm{PsNinA}$ were dependent on the size of the fragment, two shorter cDNA fragments, corresponding to nucleotides 1,879 to 2,149 and 1,964 to 2,149 , were cloned into the PEBV RNA2 vector, thereby creating the constructs pCAPE2-PsNinA253 (253 bp) and pCAPE2-PsNinA186 (186 bp), respectively (Fig. 1B). Because we had observed that the absolute number of nodules varied between experiments, PsNinA and PsNinB were included in two experiments that were set up to determine the nodulation phenotype induced upon agroinoculation with pCAPE1/pCAPE2-PsNinC, pCAPE1/ pCAPE2-PsNinA253 and pCAPE1/pCAPE2-PsNinA186. In each experiment, nine plants were agroinoculated with each construct. Combined results (Fig. 2B) indicated that decreasing the size of the PsNinA fragment resulted in an increase in nodule formation. Statistical analysis confirmed that plants inoculated with pCAPE1/pCAPE2-PsNinA186 formed significantly more nodules $(P<0.0004)$ and more pink nodules $(P<0.02)$ than plants agroinoculated with pCAPE1/pCAPE2-PsNinA. Plants inoculated with pCAPE1/ pCAPE2-PsNinA253 formed more nodules $(P<0.05)$ than plants inoculated with pCAPE1/pCAPE2-PsNinA but the difference in formation of pink nodules was not significant. Plants agroinoculated with pCAPE1/pCAPE2-PsNinC formed very few nodules and statistical analysis showed that there was no significant difference between the number of nodules $(P>0.17)$ or pink nodules $(P>0.20)$ formed on plants agroinoculated with pCAPE1/pCAPE2-PsNinA and plants agroinoculated with pCAPE1/pCAPE2-PsNinC. This result suggested that the nodulation phenotype seen upon agroinoculation of pCAPE1/pCAPE2-PsNinA and pCAPE1/ pCAPE2-PsNinC was due to silencing of PsNin.

\section{Root/shoot ratio and growth of plants agroinoculated with constructs PsNinA and PsNinB.}

To rule out the possibility that the reduction in nodulation was caused by a reduction in root size, the fresh weight of roots was measured 3 weeks after Rhizobium spp. application on all plants in the first experiment. There was no difference in the average fresh weight of roots of plants inoculated with pCAPE1/pCAPE2-PsNinA or pCAPE1/pCAPE2-PsNinB compared with plants agroinoculated with pCAPE1/pCAPE2-Con (Fig. 3). This indicated that reduction in the number of root nodules observed for plants agroinoculated with pCAPE1/
pCAPE2-PsNinA or pCAPE1/pCAPE2-PsNinB was not caused by unspecific impairment of root growth.

Inhibition of nodule formation can be expected to increase $\mathrm{N}$-starvation in plants growing under limiting $\mathrm{N}$ conditions and, thereby, lead to an increased root/shoot ratio (Davidson 1969; Levin et al. 1989). To use the root/shoot ratio as an indicator for N-starvation, shoot fresh weights were measured and root/shoot ratios calculated (Fig. 3). Plants agroinoculated with pCAPE1/pCAPE2-Con or a second control vector, pCAPE1/ pCAPE2-Con*, containing a 400-bp fragment of the GUS gene, both had an average root/shoot ratio of 1.5, whereas plants agroinoculated with pCAPE1/pCAPE2-PsNinA or pCAPE1/pCAPE2-PsNinB had a significantly higher average root/shoot ratio of 2.8 (Fig. 3). Phenotypically, shoot growth was reduced in plants agroinoculated with either pCAPE1/ pCAPE2-PsNinA or pCAPE1/pCAPE2-PsNinB compared with plants agroinoculated with either pCAPE1/pCAPE2-Con or pCAPE2Con* (Supplementary Fig. S2). Shoots of plants agroinoculated with pCAPE1/pCAPE2-PsNinA maintained two green fully developed leaves that were more light green due to $\mathrm{N}$-starvation than shoots with three fully developed leaves seen on plants agroinoculated with pCAPE1/pCAPE2-PsNinB. Application of nutrient medium containing $\mathrm{KNO}_{3}$ resulted in recovery of shoot growth. In general, growth on nutrient medium without $\mathrm{N}$ stressed the plants and the primary shoot died. Even plants agroinoculated with control construct did not receive any nitrogen supplement until pink nodules had developed 5 to 6 weeks after germination. Taken together, the significantly altered root/shoot ratios of plants agroinoculated with pCAPE1/pCAPE2-PsNinA or pCAPE1/pCAPE2-PsNinB and the phenotypes described above were consistent with an inhibition of N-fixation as would be expected by interference with nodule development.

In wild-type $P$. sativum and other nodule-forming plants, application of symbiotic bacteria results in root hair curling and formation of infection threads whereas nin mutants are characterized by excessive root hair curling and inability to form infection threads (Borisov et al. 2003; Marsh et al. 2007; Schauser et al. 1999). Root hairs of plants agroinoculated with pCAPE1/pCAPE2-PsNinA or pCAPE1/pCAPE2-Con were

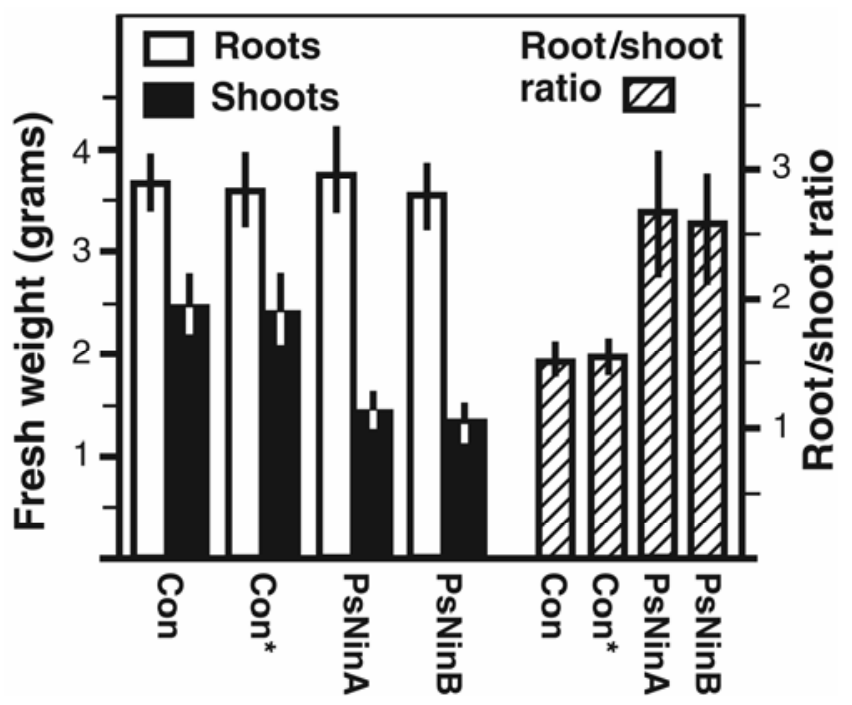

Fig. 3. Fresh weight of roots and shoots and root/shoot ratios. Roots and shoots of plants agroinoculated with pCAPE1/pCAPE2-Con (Con), pCAPE1/pCAPE2-Con* (Con*), pCAPE1/pCAPE2-PsNinA (PsNinA), and pCAPE1/pCAPE2-PsNinB (PsNinB) were weighed 3 weeks after Rhizobium spp. application. Average root weight (black columns), shoot weight (white columns), and root/shoot ratios (hatched columns) of plants from two experiments; bars represent $95 \%$ confidence intervals. 
inspected to determine whether agroinoculation with pCAPE1/pCAPE2-PsNinA would give rise to a phenotype reminiscent of the nin mutant phenotype. Few infection threads were observed in plants agroinoculated with pCAPE1/ pCAPE2-PsNinA and many root hairs displayed excessive curling.

\section{Quantification of viral RNA.}

As mentioned above, it was unexpected that agroinoculation with pCAPE1/pCAPE2-PsNinA and pCAPE1/pCAPE2PsNinB induced different nodulation phenotypes. The PsNin fragments PsNinA and PsNinB were of equal size and, to our knowledge, there are no previous examples that equal-sized fragments of the same gene causing significantly different phenotypes when inoculated as part of a VIGS vector. Nodulation was reduced to the same extent after agroinoculation with pCAPE1/pCAPE2-PsNinA and pCAPE1/pCAPE2-PsNinC (Fig. 2B). Therefore, targeting of a region encoding a nonconserved region of PsNIN appeared to have the same effect as targeting the PsNinA region encoding the conserved RWP-RK domain, which hypothetically could cause silencing of transcripts from several Nin gene family members. Instead, the PSNinB insert may affect virus accumulation and spread, leading to differences in the induction of silencing. In order to compare the amount of RNA2 derived from pCAPE1/ pCAPE2-PsNinA and pCAPE1/pCAPE2-PsNinB agroinoculation, RNA2 was quantified by real-time reverse-transcriptase polymerase chain reaction (RT-PCR) on total RNA extracted from whole roots of five plants 5 days after Rhizobium spp. application in two experiments (Fig. 4A). Results indicated that RNA2 derived from pCAPE2-PsNinA had accumulated to a significantly higher level than RNA2 derived from pCAPE2PsNinB $(P<0.05)$. This may explain the difference in nodulation phenotype in plants agroinoculated with pCAPE1/ pCAPE2-PsNinA and pCAPE1/pCAPE2-PsNinB, respectively. The secondary structure of the inserts may affect both virus accumulation and induction of silencing; however, analysis of the PsNin fragments PsNinA and PsNinB using the RNA secondary structure prediction program at GeneBee Molecular

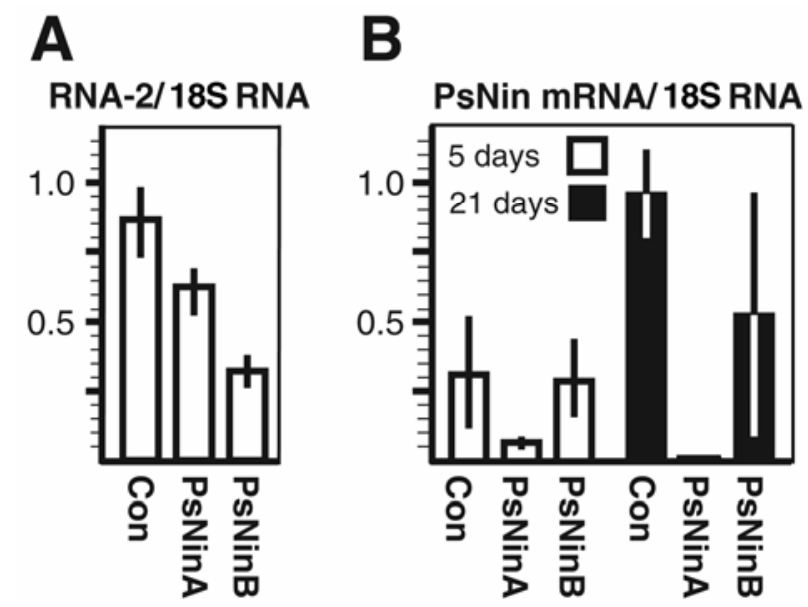

Fig. 4. Relative quantification of Pea early browning virus (PEBV) RNA2 and PsNin mRNA by real-time reverse-transcriptase polymerase chain reaction. Relative levels of PEBV RNA2, PsNin mRNA, and Pisum sativum 18S RNA were determined by amplification of cDNA derived from total RNA extracted from roots agroinoculated with pCAPE1/pCAPE2-Con (Con), pCAPE1/pCAPE2-PsNinA (PsNinA), or pCAPE1/pCAPE2PsNinB (PsNinB). Columns display data combined from two experiments. A, Relative amounts of PEBV RNA2 5 days after Rhizobium spp. application. B, Relative amounts of PsNin mRNA 5 and 21 days after Rhizobium spp. application normalized to relative amounts of $18 \mathrm{~S}$ RNA. Bars represent the $95 \%$ confidence intervals.
Biology Server did not produce conclusive results. An siRNA scan (Xu et al. 2006) predicted that fragments PsNinA and PsNinB each would generate 33 efficient siRNAs, whereas PsNinC would generate 30 efficient siRNAs.

\section{Quantification of PsNin transcript.}

In order to determine whether the effect on nodulation was reflected in alterations of PsNin transcript levels, total RNA was extracted from whole roots of 5 to 10 plants agroinoculated with pCAPE1/pCAPE2-PsNinA, pCAPE1/pCAPE2PsNinB, or pCAPE1/pCAPE2-Con. Roots were harvested either 5 or 21 days after Rhizobium spp. application. In control plants, PsNin transcription was induced and white nodules were developing 5 days after Rhizobium spp. application whereas, 21 days after Rhizobium spp. application, PsNin transcript levels were high, probably due to expression in mature pink nodules. Therefore, the quantification 5 days after Rhizobium spp. application was included to estimate the direct effect of VIGS on PsNin transcript levels, whereas the PsNin transcript levels 21 days after Rhizobium spp. application reflect the lack or reduction of developing and mature nodules. PsNin transcripts were quantified by real-time RT-PCR using primers amplifying a region $5^{\prime}$ to the PsNin fragments PsNinA and PsNinB. For plants agroinoculated with pCAPE1/pCAPE2-PsNinA, PsNin transcript levels were significantly lower than in plants agroinoculated with pCAPE1/pCAPE2-Con at both 5 and 21 days after Rhizobium spp. application (Fig. 4B). For plants agroinoculated with pCAPE1/pCAPE2-PsNinB, PsNin transcripts levels were similar to controls 5 days after Rhizobium spp. application but reduced when measured 21 days after Rhizobium spp. application. Thus, downregulation of PsNin transcript appeared to occur earlier in the pCAPE2-NinA-inoculated plants than in pCAPE2-NinB-inoculated plants, correlating with the different stages at which nodule formation was inhibited by the two constructs.

\section{DISCUSSION}

The main purpose of the present study was to determine whether VIGS could affect expression of a nodulin gene to cause a change in nodulation phenotype in $P$. sativum. To address this, PsNin gene transcripts were targeted for degradation through agroinoculation with the PEBV cDNA vector carrying fragments of PsNin. When silencing was evident by bleaching of leaves in a set of reference plants agroinoculated with pCAPE1/pCAPE2-PDS, Rhizobium culture was applied to the growth medium (Fig. 1A). Three fragments of PsNin were tested and, of these, PsNinA and PsNinC (Fig. 1B) almost completely inhibited nodulation while the third fragment, PsNinB, caused a significant reduction in nodulation (Fig. 2). These results indicated that silencing induced by PEBV in roots was more efficient than in green parts, where only a fraction of the flowers were affected upon silencing of UNIFOLIATA and bleaching occurred in patches on leaves upon silencing of PSPDS (Constantin et al. 2004). An explanation could be that PEBV is a nematode-transmitted virus (Robinson 2003) and transmission of the virus relies on root infection. However, silencing in roots by TRV, which is also nematode transmitted, was reported to be less efficient in roots compared with leaves (Bhattarai et al. 2007; Valentine et al. 2004).

The fragments PsNinA, PsNinB, and PsNinC were of similar size (430 to $436 \mathrm{bp}$ ). To our knowledge, it has not been observed previously that VIGS induced by fragments of similar size from the same gene result in different phenotypes. In the experiments where more than one fragment was tested, no differences were reported in phenotype or transcript reduction (Kim et al. 2005; Kim et al. 2006). Therefore, the difference 
between fragments PsNinA and PsNinB were analyzed in more detail. We found that the difference in nodulation phenotype (Fig. 2A) developing after agroinoculation with pCAPE1/ pCAPE2-PsNinA and pCAPE1/pCAPE2-PsNinB correlated with the effect on PsNin transcript levels (Fig. 4B). The fragment of PsNin carried by pCAPE2-PsNinA encodes a region of NIN which is conserved in proteins encoded by the Nin gene family. Therefore, it was possible that off-target silencing of genes belonging to this family caused the strong effect on nodulation observed after agroinoculation by pCAPE1/ pCAPE2-PsNinA. However, this interpretation was not supported by results from agroinoculation with pCAPE1/pCAPE2PsNinC. The 433-bp PsNinC insert does not encode a region of PsNIN that is conserved in genes encoded by other members of the Nin gene family (Schauser et al. 2005), and agroinoculation with pCAPE1/pCAPE2-PsNinC had an effect on nodulation comparable to agroinoculation with pCAPE1/ pCAPE2-PsNinA. An siRNA Scan predicted that fragments PsNinA and PsNinB would both generate 33 efficient siRNAs, but other factors such as target accessibility may influence silencing efficiency ( $\mathrm{Xu}$ et al. 2006). It was not possible to make conclusions concerning off-targets based on bioinformatic analysis because of the limited sequence information on $P$. sativum.

The observations that virus vectors carrying small inserts with inverted repeats are more efficient in inducing silencing (Lacomme et al. 2003) and that viral RNA with imperfect duplexes may be the predominant target of DICER (Molnár et al. 2005) suggest that differences in the secondary structure of the PsNin fragments could explain the observed difference in nodulation of plants agroinoculated with pCAPE1/pCAPE2PsNinA and pCAPE1/pCAPE2-PsNinB. However, we could not identify obvious differences in secondary structure of the PsNinA and PsNinB fragments using the RNA secondary structure prediction program at GeneBee Molecular Biology Server. Instead, we found that PEBV RNA2 accumulated to significantly higher levels in plants agroinoculated with pCAPE1/pCAPE2-PsNinA than in plants agroinoculated with pCAPE1/pCAPE2-PsNinB (Fig. 4A), and this was correlated with a larger reduction in PSNin transcripts 5 days after Rhizobium spp. application (Fig. 4B). A similar correlation was observed in tomato inoculated with a TRV VIGS vector carrying a fragment of PDS (Rotenberg et al. 2006). This would suggest that increased virus accumulation improved VIGS and that the virus RNA was better protected from silencing than the target mRNA (Ding and Voinnet 2007). The quantification of PEBV RNA2 showed that, in plants agroinoculated with pCAPE1/ pCAPE2-Con, RNA2 accumulated to even higher levels than in plants agroinoculated with pCAPE1/pCAPE2-PsNinA (Fig. 4A). The difference in RNA2 accumulation cannot be explained by differences in the length of the heterologous inserts in pCAPE2-Con, pCAPE2-PsNinA, and pCAPE2-PsNinB and, as in other systems, there does not seem to be a simple correlation between fragment length, virus accumulation, and silencing phenotype (Bruun-Rasmussen et al. 2007; Thomas et al. 2001).

Nin transcripts are highly expressed in developing nodules, suggesting that NIN is required not only at nodule inception but also at later stages of nodule development (Borisov et al. 2003; Schauser et al. 1999). Possibly, the weaker phenotype observed after agroinoculation with pCAPE1/pCAPE2-PsNinB, with a 45 to $70 \%$ reduction in visible nodules, can be used for uncovering later functions of NIN. With our present knowledge, we cannot accurately predict the silencing efficiency of a VIGS construct; however, reducing the length of the insert from $430 \mathrm{bp}$ in pCAPE2-PsNINA to $253 \mathrm{bp}$ in pCAPE2NinA253 and 186 bp in pCAPE2-PsNinA186 weakened the phenotype (Fig. 2B). Still, the total number of nodules was significantly $(P<0.01)$ lower after agroinoculation with pCAPE1/pCAPE2-PsNin186 than after agroinoculation with pCAPE1/pCAPE2-PsNinB, demonstrating that factors other than length were important.

The plants that were agroinoculated with pCAPE1/pCAPE2PsNin constructs showed clear signs of $\mathrm{N}$ starvation, reflected in significantly increased root/shoot ratios compared with plants agroinoculated with pCAPE1/pCAPE2-Con (Fig. 3) (Davidson 1969; Levin et al. 1989). The plants inoculated with pCAPE1/ pCAPE2-PsNinB developed nodules and the plants appeared equally $\mathrm{N}$ starved as plants inoculated with pCAPE1/pCAPE2PsNinA, judged from the root/shoot ratio (Fig. 3). However, plants agroinoculated with pCAPE1/pCAPE2-PsNinA maintained only two green leaves compared with three in plants agroinoculated with pCAPE1/pCAPE2-PsNinB, suggesting that the nodules on these plants did provide some $\mathrm{N}$.

Though we cannot explain why agroinoculation with pCAPE1/pCAPE2-PsNinA and pCAPE1/pCAPE2-PsNinC induces a stronger phenotype than agroinoculation with pCAPE1/ pCAPE2-PsNinB, our results suggested that it may be possible to modulate silencing strength by choice of fragment as well as by altering the fragment length. Here, agroinoculation with pCAPE1/pCAPE2-PsNinA and pCAPE1/pCAPE2-PsNinC gave silencing phenotypes resembling the mutant sym35 non-nodulating phenotype (Borisov et al. 2003) whereas agroinoculation with pCAPE1/pCAPE2-PsNinB, pCAPE1/pCAPE2-PsNin186, and pCAPE1/pCAPE2-PsNin253 resulted in a 'leaky' phenotype. An advantage provided by VIGS is the possibility to analyze the effect of targeting a single gene in the same genetic background, in contrast to mutant studies where backcrossing may be needed to eliminate other mutations or T-DNA inserts. In P. sativum, application of VIGS can facilitate studies of gene function despite the large genome and recalcitrance to transformation, and makes it possible to take advantage of the knowledge generated previously on nodulation in this species.

\section{MATERIALS AND METHODS}

\section{Plants growth and bacterial strains.}

P. sativum cv. Dark Skinned Perfection (Dæhnfeldt, Odense, Denmark) plants were used in all experiments. To grow plants under sterile conditions, seed were surface sterilized in $1.5 \%$ hypochlorite for $20 \mathrm{~min}$, rinsed in sterile water, and placed for germination for 2 days in darkness at room temperature. Germinated seed were transferred to clay granules (Fiboklinker from Maxit, Risskov, Denmark) that had been autoclaved in a semiclosed growth system composed of two Magenta boxes (Sigma, Brøndby, Denmark), with the lower box containing Nfree Jensen medium (van Brussel et al. 1982) and a cotton wick from the top box to the bottom box. Plants were grown in climate chambers with $16-$ and 8 -h photoperiod at $200 \mu \mathrm{E}$, $20^{\circ} \mathrm{C}$, and $75 \%$ humidity.

Plants were agroinoculated 2 weeks after germination as described by Constantin and associates (2004), except that gentamicin (Sigma) at $25 \mu \mathrm{g} \mathrm{ml}^{-1}$ replaced rifampicin in the selection media for the bacteria. R. leguminosarum bv. viceae strain 248 (Josey et al. 1979) was grown to an optical density at 660 of 0.3 to 0.5 in YMB medium (Handberg et al. 1994). For Rhizobium spp. inoculation, the culture was diluted 1:19 in Jensen medium and $150 \mathrm{ml}$ was applied to the upper magenta box. Medium containing $\mathrm{N}$ consisted of Jensen medium with 1 $\mathrm{mM} \mathrm{KNO}_{3}$.

Secondary lateral roots were inspected in the root hair growth zone using a Leitz Laborlux S Microscope (Leica, Herlev, Denmark) and photographs were taken using Nikon Coolpix 4500 (Nikon, Copenhagen, Denmark). Root fragments were col- 
lected from three plants agroinoculated with pCAPE1/pCAPE2PsNinA or pCAPE1/pCAPE2-Con 3 weeks after Rhizobium spp. application. Root sections were stained in $0.05 \%$ toluidine blue (Merck, Glostrup, Denmark).

\section{Cloning of silencing constructs.}

The binary vectors pCAPE1 and pCAPE2 containing cDNA of PEBV RNA1 and RNA2, respectively, were manipulated by standard cloning methods. Restriction and modifying enzymes (Roche Diagnostics, Mannheim, Germany and New England Biolabs, Ipswich, MA, U.S.A) and Taq DNA polymerase (GibcoBRL, Roskilde, Denmark) were used according to the manufacturers' instructions. PCR products were cloned using TOPO-technology (Invitrogen, Taastrup, Denmark). Oligonucleotide primers were provided by MWG (Ebersberg, Germany). To target PsNin for VIGS, fragments of PsNin cDNA from $P$. sativum cv. Finale (AJ493066) (Borisov et al. 2003) were inserted into pCAPE2-PDS (Constantin et al. 2004), replacing the $P D S$ gene fragment. The PsNinA fragment of 430 bp (nucleotides 1,720 to 2,149) was amplified by PCR using forward primer $5^{\prime}$-cactagTTCAGTCTTTTCAGA and reverse primer 5'-GCTGCAGTGCTCAACTCTGGA and inserted as a SpeI/Pst I fragment generating pCAPE2-PsNinA. The PsNinA253 fragment of $253 \mathrm{bp}$ (nucleotides 1,897 to 2,149) was amplified by PCR using forward primer 5 '-ccatggCATAA GCTTGCCTGTTCTAA and reverse primer 5'-GCTGCAGTG CTCAACTCTGGA and inserted as an NcoI/PstI fragment generating pCAPE2-PsNinA253. The PsNinA186 fragment of $186 \mathrm{bp}$ (nucleotides 1,964 to 2,149) was amplified by PCR using forward primer 5 '-ccatggGTATGTCCTACAACTCTGA and reverse primer $5^{\prime}$-GCTGCAGTGCTCAACTCTGGA and inserted as an NcoI/PstI fragment generating pCAPE2PsNinA186. The PsNinB fragment of $436 \mathrm{bp}$ (nucleotides 2,212 to 2,647) was excised as an NcoI/Pst I fragment and inserted into pCAPE2-PDS generating pCAPE2-PsNinB. The PsNinC fragment of $433 \mathrm{bp}$ (nucleotides 106 to 538) was amplified by PCR using forward primer 5'-gactagtctGGAATATGGTGGT GGGATAGTGGA and reverse primer 5 '-gaagatcttCTGCTGA AGATAATGAATTGCCGA and inserted as an SpeI/BglII fragment generating pCAPE2-PsNinC. Two control constructs were used in the experiments: pCAPE2-Con (Constantin et al. 2004) and pCAPE2-Con* containing a 400-bp fragment of the GUS gene amplified with forward primer 5 '-ccatggTTTAACGA TCAGTTCGCCGATGCAGAT and reverse primer 5'-ctgcagCA CCCAGGTGTTCGGCGTGGTGTA and inserted as an NcoI/ $P s t \mathrm{I}$ fragment into pCAPE2-PDS replacing the PDS gene.

The three fragments PsNinA, PsNinB, and PsNinC all were analyzed by the siRNA Scan program (Xu et al. 2007) to determine the number of efficient siRNAs predicted from each fragment.

\section{Real-time RT-PCR.}

Samples were collected from five randomly selected plants per treatment per experiment. For each sample, total RNA was extracted from $100 \mathrm{mg}$ of root tissue using RNeasy plant mini kit (Qiagen, Hilden, Germany) with on-column DNase treatment following the manufacturer's recommendations. An additional DNase treatment was performed for $15 \mathrm{~min}$ at $37^{\circ} \mathrm{C}$ with $0.8 \mathrm{U}$ of RQ1 RNase-free DNase (Promega Corp., Madison, WI, U.S.A.) per $40 \mu \mathrm{l}$ of RNA eluate. The DNase reaction was terminated by addition of RQ1 DNase Stop Solution (Promega Corp.) and subsequent incubation for $10 \mathrm{~min}$ at $65^{\circ} \mathrm{C}$. RNA concentration was measured on a Nanodrop ND1000 Spectrophotometer (Saveen Werner, Malmö, Sweden) and $0.5 \mu \mathrm{g}$ was used for first-strand cDNA synthesis with random hexamer primers for the RT reaction, as described previously (Constantin et al. 2004).
For relative quantification of RNA by real-time RT-PCR, 18S (U43011) primers 18SFW 5'-GACTACGTCCCTGCCCT TTG (nucleotides 1,571 to 1,590 ) and 18SRV 5'-AACACTT CACCGGACCATTCA (nucleotides 1,618 to 1,638), PEBV RNA2 (X78455) primers PE2FW 5'-TCGGTTTGCTGACCT ACTGG (nucleotides 461 to 480) and PE2RV 5'-CCACTTC GACCCAAGCACTA (nucleotides 566 to 585), and PsNin (AJ493066) primers NinFW 5'-GGATTCCAAGAAGCTTGC AG (nucleotides 1,094 to 1,113) and NinRV 5'-CTACACTGG CTCTGCAGCTG (nucleotides 1,282 to 1,301) were designed using Primer3 (Rozen and Skaletsky 2000). Real-time PCR was performed using the 7500 Real Time PCR System (Applied Biosystems, Naerum, Denmark). Each $20 \mu \mathrm{l}$ of PCR reaction contained $4 \mu \mathrm{l}$ of RT reaction, $12 \mu \mathrm{l}$ of SYBR Green Master Mix (Applied Biosystems), and $500 \mathrm{nM}$ each primer. Samples were heated to $95^{\circ} \mathrm{C}$ for $10 \mathrm{~min}$, followed by $40 \mathrm{cy}$ cles of $15 \mathrm{~s}$ at $95^{\circ} \mathrm{C}$ and $1 \mathrm{~min}$ at $60^{\circ} \mathrm{C}$. Each RT reaction was analyzed in duplicate. The 7500 System Software program calculated relative amounts of RNA based on PCR cycle threshold values obtained from a fourfold dilution series of a standard RT sample from a plant inoculated with pCAPE1/pCAPE2Con. Calculated relative amounts of target gene RNA were normalized by calculated relative amounts of $18 \mathrm{~S}$ RNA.

\section{Statistical analysis.}

Data were analyzed by InStat 3.0 software (GraphPad Software, San Diego, CA, U.S.A.). The nonparametric MannWhitney test was applied to analysis of nodule numbers presented in Figure 2. Other statistical analyses were performed using an unpaired $t$ test. Data without a normal distribution were transformed by converting to a natural logarithm.

\section{ACKNOWLEDGMENTS}

We thank B. Olsen and M. Læssøe Steffensen for expert technical assistance with laboratory work and maintenance of plants in the greenhouse. This work was supported by the Danish Agricultural and Veterinary Research Council (grant no. 53-00-0330 to O. S. Lund and grant no. 23-030118 to M. Grønlund) and by The Danish National Research Foundation (Ph.D. stipend to G. Constantin).

\section{LITERATURE CITED}

Barbulova, A., Rogato, A., D’Apuzzo, E., Omrane, S., and Chiurazzi, M. 2007. Differential effects of combined N sources on early steps of the Nod factor-dependent transduction pathway in Lotus japonicus. Mol. Plant-Microbe Interact. 20:994-1003.

Bennett, M. D., and Leitch, I. J. 2004 Plant DNA C-values database (release 3.0, Dec. 2004) Royal Botanic Gardens, Kew, U.K.

Bhattarai, K. K., Li, Q., Liu, Y, Dinesh-Kumar, S. P., and Kaloshian, I. 2007. The MI-1-mediated pest resistance requires Hsp90 and Sgt1. Plant Physiol. 144:312-323.

Borisov, A. Y., Madsen, L. H., Tsyganov, V. E., Umehara, Y., Voroshilova, V. A., Batagov, A. O., Sandal, N, Mortensen, A., Schauser, L., Ellis, N., Tikhonovich, I. A., and Stougaard, J. 2003. The Sym35 gene required for root nodule development in pea is an ortholog of Nin from Lotus japonicus. Plant Physiol. 131:1009-1017.

Bruun-Rasmussen, M., Madsen C. T., Jessing, S., and Albrechtsen, M. 2007. Stability of Barley stripe mosaic virus-induced gene silencing in barley. Mol. Plant-Microbe Interact. 20:1323-1331.

Burch-Smith, T. M., Anderson, J. C., Martin, G. B., and Dinesh-Kumar, S. P. 2004. Applications and advantages of virus-induced gene silencing for gene function studies in plants. Plant J. 39:734-746.

Constantin, G. D., Krath, B. N., MacFarlane, S. A., Nicolaisen, M., Johansen, I. E., and Lund, O. S. 2004. Virus-induced gene silencing as a tool for functional genomics in a legume species. Plant J. 40:622-631.

Davidson, R. L. 1969. Effects of soil nutrients and moisture on root/shoot ratios in Lolium perenne L. and Trifolium repens L. Ann. Bot. 33:571577.

Ding, S.-W., and Voinnet, O. 2007. Antiviral immunity directed by small RNAs. Cell 130:413-426.

Duc, G., and Messager, A. 1989. Mutagenesis of pea (Pisum sativum L.) 
and the isolation of mutants for nodulation and nitrogen fixation. Plant Sci. 60:207-213.

Handberg, K., Stiller, J., Thykjaer, T., and Stougaard, J. 1994. Agrobacterium-mediated transformation of the diploid legume Lotus japonicus. Pages 119-129 in: Cell Biology: A Laboratory Handbook. Academic Press, New York.

Josey, D. P., Beynon, J. L., Johnston, A. W. B., and Beringer, J. E. 1979. Strain identification in Rhizobium using intrinsic antibiotic resistance. J. Appl. Bacteriol. 46:343-350.

Kim, M., Lim, J.-H., Ahn, C. S., Park, K., Kim, G. T., Kim, W. T., and Pai, H.-S. 2006. Mitochondria-associated hexokinases play a role in the control of programmed cell death in Nicotiana benthamiana. Plant Cell 18:2341-2355.

Kim, Y.-K., Lee, J.-Y., Cho, H. S., Lee, S. S., Ha, H. J., Kim, S., Choi, D., and Pai, H.-S. 2005. Inactivation of organellar glutamyl- and seryltRNA synthetases leads to developmental arrest of chloroplasts and mitochondria in higher plants. J. Biol. Chem. 280:37098-37106.

Krejcí, P., Matusková, P., Hanácek, P., Reinöhl, V., and Procházka, S. 2007. The transformation of pea (Pisum sativum L.): Applicable methods of Agrobacterium tumefaciens-mediated gene transfer. Acta Physiol. Plant. 29:157-163.

Lacomme, C., Hrubikova, K., and Hein, I. 2003. Enhancement of virusinduced gene silencing through viral-based production of inverted-repeats. Plant J. 34:543-553.

Levin, S. A., Mooney, H. A., and Field, C. 1989. The dependence of plant root: Shoot ratios on internal nitrogen concentration. Ann. Bot. 64:71-75.

Liu, Y., Schiff, M., Czymmek, K., Talloczy, Z., Levine., B., and DineshKumar, S. P. 2005. Autophagy regulates programmed cell death during the plant innate immune response. Cell 121:567-577.

MacFarlane, S. A., and Popovich, A. H. 2000. Efficient expression of foreign proteins in roots from tobravirus vectors. Virology 267:29-35.

Marsh, J. F., Rakocevic, A., Mitra, R. M., Brocard, L., Sun, J., Eschstruth, A., Long, S. R., Schultze, M., Ratet, P., and Oldroyd, G. E. 2007. Medicago truncatula NIN is essential for rhizobium-independent nodule organogenesis induced by autoactive calcium-calmodulin-dependent protein kinase. Plant Physiol. 144:324-335.

Molnár, A., Csorba, T., Lakatos, L., Várallyay, É., Lacomme, C., and Burgyán, J. 2005. Plant virus-derived small interfering RNAs originate predominantly from highly structured single-stranded viral RNAs. J. Virol. 79:7812-7818.

Ott, T., van Dongen, J. T., Günther, C., Krusell, L., Desbrosses, G., Vigeolas, H., Bock, V., Czechowski T., Geigenberger, P., and Udvardi, M. K. 2005. Symbiotic leghemoglobins are crucial for nitrogen fixation in legume root nodules but not for general plant growth and development. Curr. Biol. 15:531-535.

Robinson, D. 2003. Tobacco rattle virus. In: Descriptions of Plant Viruses No. 398. Association of Applied Biologists, Warwick, U.K.

Rotenberg, D., Thompson, T. S., German, T. L., and Willis, D. K. 2006.
Methods for effective real-time RT-PCR analysis of virus-induced gene silencing. J. Virol. Methods 138:49-59.

Rowland, O., Ludwig, A. A., Merrick, C. J., Baillieul, F., Tracy, F. E., Durrant, W. E., Fritz-Laylin, L., Nekrasov, V., Sjölander, K., Yoshioka, H. and Jones, J. D. 2005. Functional analysis of Avr $9 / C f-9$ Rapidly Elicited genes identifies a protein kinase, ACIK1, that is essential for full Cf-9dependent disease resistance in tomato. Plant Cell 17:295-310.

Rozen, S., and Skaletsky, H. 2000. Primer3 on the WWW for general users and for biologist programmers. Methods Mol. Biol. 132:365-386.

Schauser, L., Roussis, A., Stiller, J., and Stougaard, J. 1999. A plant regulator controlling development of symbiotic root nodules. Nature 402:191-195.

Schauser, L., Wieloch, W., and Stougaard, J. 2005. Evolution of NIN-like proteins in Arabidopsis, rice and Lotus japonicus. J. Mol. Evol. 60:229237.

Spaink, H. P. 2000. Root nodulation and infection factors produced by rhizobial bacteria. Annu. Rev. Microbiol. 54:257-288.

Thomas, C. L., Jones, L., Baulcombe, D. C, and Maule, A. J. 2001. Size constraints for targeting post-transcriptional gene silencing and for RNA-directed methylation in Nicotiana benthamiana using a Potato virus $X$ vector. Plant J. 25:417-425.

Tirichine, L., Sandal, N., Madsen, L. H., Radutoiu, S., Albrektsen, A. S., Sato, S., Asamizu, E., Tabata, S., and Stougaard, J. 2007. A gain-offunction mutation in a cytokinin receptor triggers spontaneous root nodule organogenesis. Science 315:104-107.

Valentine, T., Shaw, J., Blok, V. C., Phillips, M. S., Oparka, K. J., and Lacomme, C. 2004. Efficient virus-induced gene silencing in roots using a modified tobacco rattle virus vector. Plant Physiol. 136:3999-4009.

van Brussel, A. A. N., Tak, T., Wetselaar, A., Pees, E., and Wijffelman, C. A. 1982. Small Leguminosae plants as test plants for nodulation of Rhizobium leguminosarum and other Rhizobia and Agrobacteria harbouring a leguminosarum Sym-plasmid. Plant Sci. Lett. 27:317-325.

Xu, P., Zhang, Y., Kang, L., Roossinck, M. J., and Mysore, K. S. 2006. Computational estimation and experimental verification of off-target silencing during posttranscriptional gene silencing in plants. Plant Physiol. 142:429-440.

Zhang, C., and Ghabrial, S. A. 2006. Development of Bean pod mottle virus-based vectors for stable protein expression and sequence-specific virus-induced gene silencing in soybean. Virology 344:401-411.

\section{AUTHOR RECOMMENDED INTERNET RESOURCES}

GeneBee Molecular Biology server: www.genebee.msu.su/genebee.html Samuel Roberts Noble Foundation's SiRNA Scan: bioinfo2.noble.org/rnaiscan.htm

Association of Applied Biologists, Descriptions of Plant Viruses website: www.dpvweb.net/dpv/index.php 